\title{
Cognition and Cooperation in Advanced Wireless and Mobile Information Systems
}

\author{
Chao Chen, ${ }^{1}$ Carlos Pomalaza-Ráez, ${ }^{2}$ Yunfei Chen, ${ }^{3}$ Kun Xie, ${ }^{4}$ and Tricha Anjali ${ }^{5}$ \\ ${ }^{1}$ Department of Electrical and Computer Engineering, Indiana University-Purdue University Fort Wayne, Fort Wayne, IN, USA \\ ${ }^{2}$ Center for Wireless Communications, University of Oulu, 90570 Oulu, Finland \\ ${ }^{3}$ School of Engineering, University of Warwick, Coventry CV4 7AL, UK \\ ${ }^{4}$ College of Computer Science and Electronic Engineering, Hunan University, Changsha, China \\ ${ }^{5}$ International Institute of Information Technology, Bangalore, India
}

Correspondence should be addressed to Chao Chen; chenc@ipfw.edu

Received 18 August 2016; Accepted 18 August 2016

Copyright (C) 2016 Chao Chen et al. This is an open access article distributed under the Creative Commons Attribution License, which permits unrestricted use, distribution, and reproduction in any medium, provided the original work is properly cited.

With the recent development in advanced sensing technology, processing capability, memory capacity, and battery innovations, modern wireless devices become increasingly aware of the conditions of their components and the surrounding environment. Based on learning results, a cognitive wireless device is able to reconfigure its operation dynamically and allocate resources efficiently to improve performance. In addition, cooperation among networked peer devices enables them to make intelligent adaptations based on the aggregated learning results to optimize the whole network performance.

A cognitive radio is an intelligent radio that can detect available frequency bands in its vicinity and reconfigure its transceiver parameters adaptively. Several of the selected papers investigate algorithms such as spectrum sensing, access control, and interference suppression that allow secondary users (SUs) to exploit the transmission opportunities in the primary network. S. S. Ali et al. propose new spectrum sensing methods in a multiantenna setting by weighting all eigenvalues of the covariance matrix, with the goal of detecting the existence of primary users (PUs) more effectively. SUs can choose one of few proposed weighting schemes depending on what prior information about PU is available. When a PU-vacant channel is detected in a cognitive radio network, traffic from SUs needs to be regulated to allow multiple cognitive radios to coexist in the same spectrum band. Y. Zhao et al. propose an access control scheme for prioritized traffic from SUs. They use a discrete-time queuing model to analyze the system performance and derive a dynamic access threshold for the SU packets with lower priority according to actual network status. D. Zhang et al. develop a rankconstrained beamforming algorithm for underlay multipleinput multiple-output (MIMO) cognitive radio networks. The proposed design aligns the interference caused by SUs into a reduced-dimensional subspace. At the same time, the PUs' receivers select the optimal precoding and receiving beamforming matrices to minimize the dimension of the interference.

Cooperative relaying can improve throughput and energy efficiency in wireless communications through additional spatial and spectrum diversity at the relay node. N. Cao et al. compare two strategies of cooperative relay, that is, decodeand-forward relaying and amplify-and-forward relaying, and their outage performance in cognitive radio systems. They propose the concept of rate decaying factor and use it to derive the SUs maximum SNR of these two relay strategies in a PU-occupied channel. Y. Yao et al. consider energyharvesting relay nodes that can transform received RF signal into stored energy. In such systems, the sender first allocates its transmission power to simultaneously transmit information and transfer power to the relay, which then forwards the information to the destination using harvested energy. Through joint optimization of the power splitting and time switching ratios, the network throughput is maximized. 
Besides those, the paper by M. Radenkovic addresses the problem of "cognitive privacy" in the mobile social world. An open-source distributed virtual platform called cognitive privacy framework is proposed, in which each personal cloud may decide on the fly to use different transmission network for different types of data and privacy requirements.

These papers are excellent examples of how cognition and cooperation in various wireless and mobile systems can improve system performance and user experience. By compiling these papers, we hope to encourage our readers and researchers to apply cognitive and cooperative principles in designing future wireless and mobile information systems.

Chao Chen

Carlos Pomalaza-Ráez

Yunfei Chen

Kun Xie

Tricha Anjali 

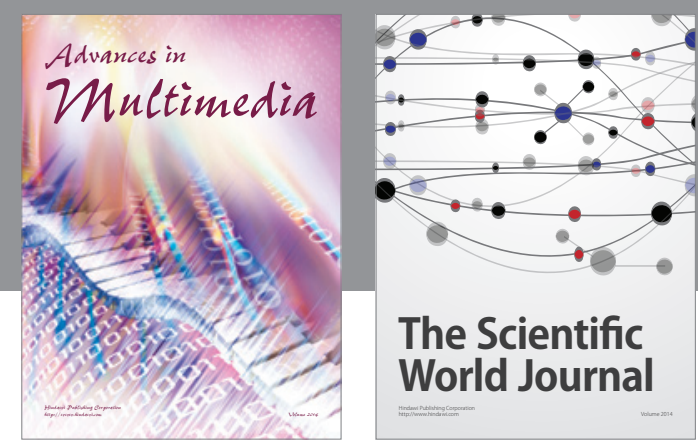

The Scientific World Journal
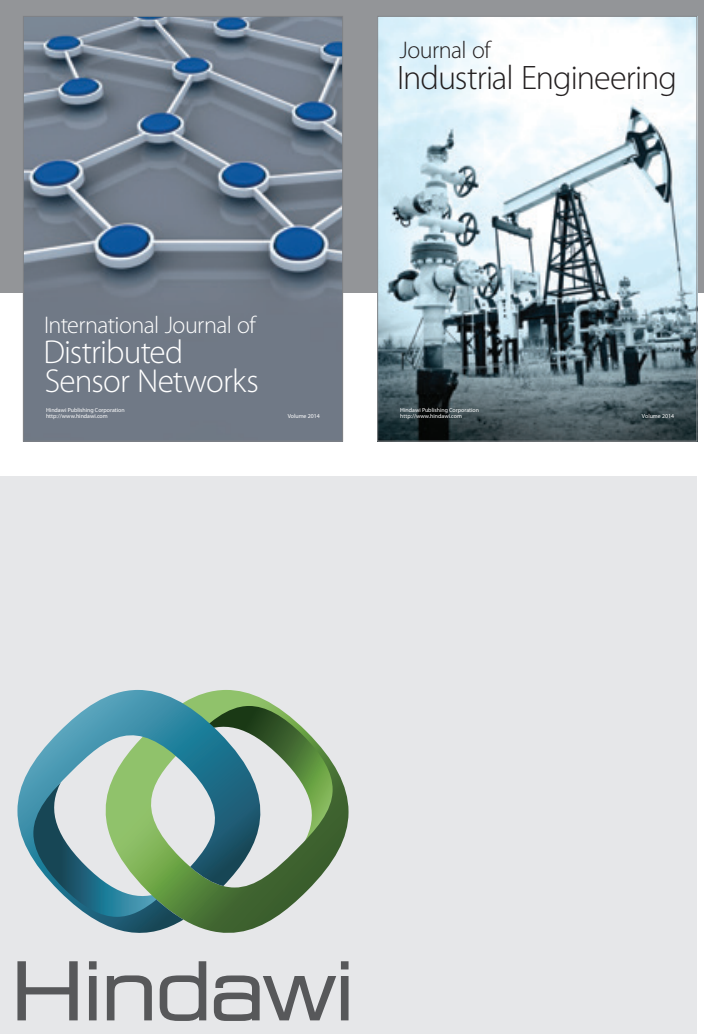

Submit your manuscripts at

http://www.hindawi.com

\section{Computer Networks} and Communications
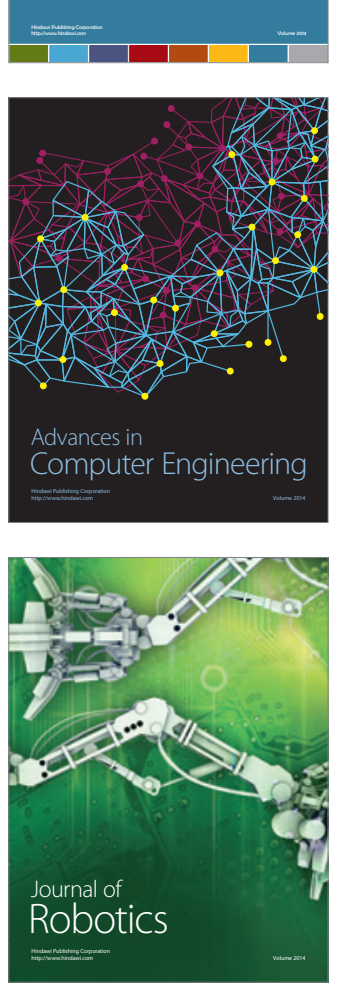
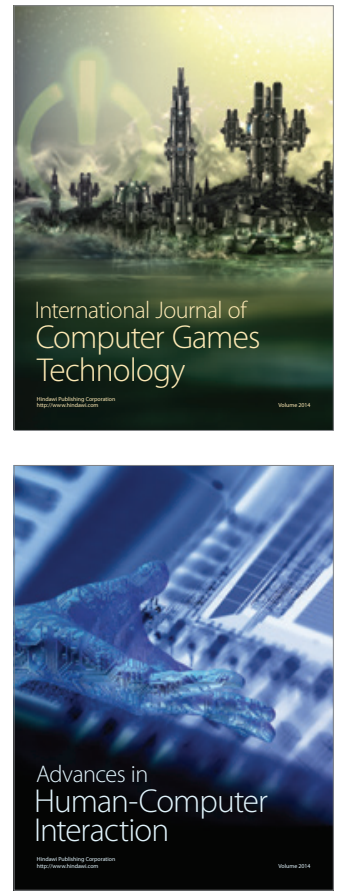
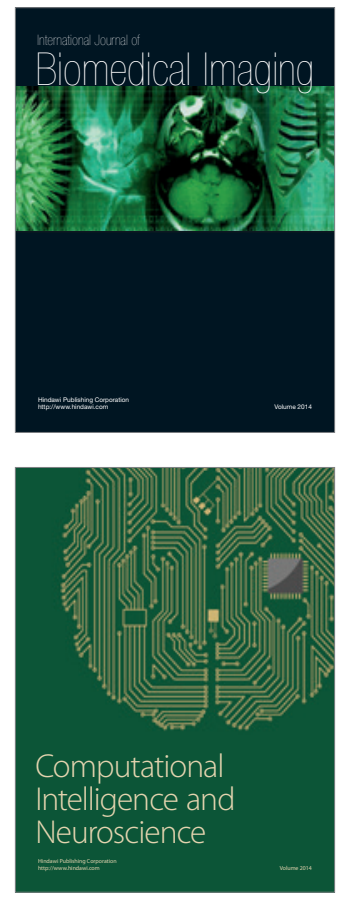
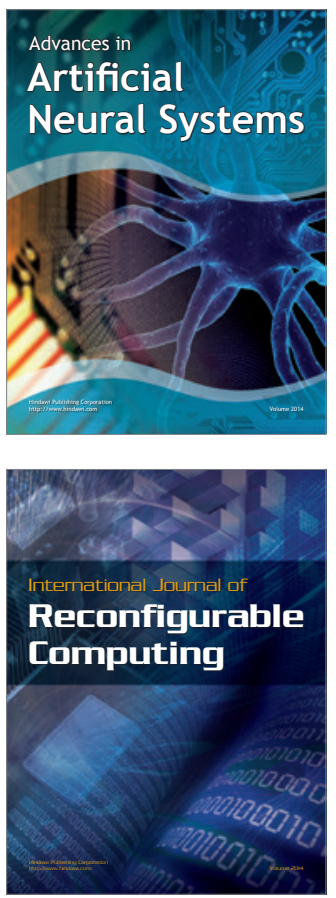
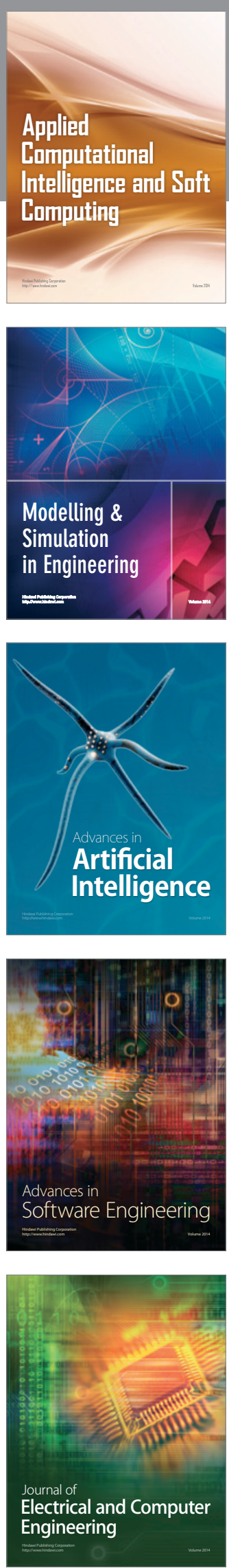